\title{
Power Maximization for Capacitive Wireless Power Transfer with Two Transmitters and One Receiver
}

\author{
Ben Minnaert and Nobby Stevens \\ KU Leuven DRAMCO \\ Department of Electrical Engineering (ESAT) \\ Technology Campus Ghent, 9000 Ghent, Belgium \\ Email: ben.minnaert@kuleuven.be, nobby.stevens@kuleuven.be
}

\begin{abstract}
Inductive wireless power transfer from multiple transmitters to a single receiver has already been studied. However, for capacitive power transfer, an analysis of a multi transmitter setup has not yet been performed, even though useful applications are being developed for this technology. In this work, we determine the optimal solution for a capacitive wireless power transfer system in order to maximize the power transfer from two transmitter sources to a single load at the receiver side. As well the case for uncoupled as coupled transmitters is solved. We find that for the uncoupled configuration, a purely resistive load optimizes the system, whereas for the coupled configuration, a susceptance has to be inserted to maximize the power transfer. We confirm our results by circuit simulations.
\end{abstract}

\section{INTRODUCTION}

By means of the electric field, capacitive coupling allows wireless energy transfer. Applications are for example the charging of integrated circuits, biomedical devices, low-power consumer applications and electric vehicles [1]. These examples consist of a single transmitter device, supplied by a source, and a single receiver, containing the load. This configuration has already been described in detail [1]-[3].

For certain applications, a configuration with multiple transmitters and/or multiple receivers could be beneficial. For example, a large transmitter plate can charge multiple receivers at once. But also the configuration with multiple transmitters and one receiver can be of interest. An example is the charging of electric vehicles while driving: multiple transmitter plates in the road could charge a driving vehicle which contains the receiver plate [1], [5], [6]. To our knowledge, a set-up with multiple transmitters for capacitive wireless power transfer (CPT) has not yet been described.

In this work, we provide the optimal solution to maximize the power transfer from two transmitter sources to a single load at the receiver. This problem has already been solved for one transmitter and one receiver [3], and for one transmitter and two receivers [4], but not yet for a multiple transmitter CPT set-up. As well the case with uncoupled as with coupled transmitters is solved. The results are confirmed by circuit simulations in SPICE.

\section{Analytical solution}

\section{A. Circuit analysis}

Fig. 1 represents a general CPT system with two transmitters and one receiver [1], [7]. We only consider the wireless link

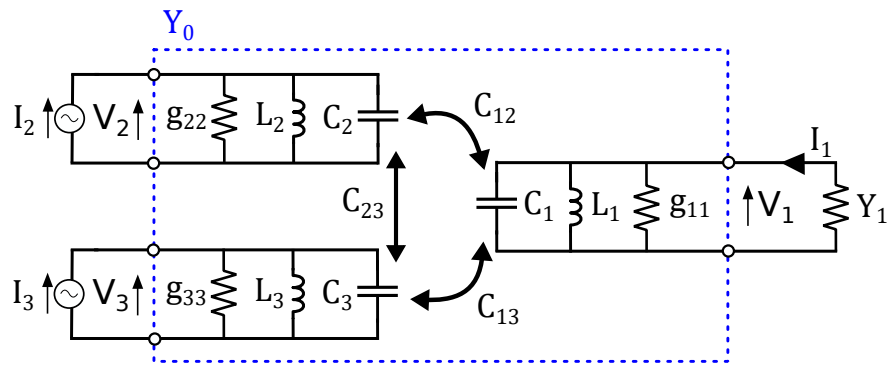

Fig. 1. A CPT circuit with two transmitters and one receiver can be represented by a three-port network, characterized by its admittance matrix $\boldsymbol{Y}_{\mathbf{0}}$, indicated by the dashed rectangle.

itself and do not take into account the remote electronics. The transmitters are supplied by the sinusoidal current sources, represented by the peak current phasors $I_{2}$ and $I_{3}$. We assume both transmitters operate at the same angular frequency $\omega_{0}$. The conductances $g_{11}, g_{22}$ and $g_{33}$ represent the resistive losses in the circuit. The load of the receiver is given by $Y_{1}=G_{1}+j B_{1}$, with $G_{1}$ and $B_{1}$ the load conductance and susceptance, respectively.

The capacitive wireless link can be represented by coupled capacitances $C_{1}, C_{2}$ and $C_{3}$ [4], [7]. Power transfer is realized from the transmitter capacitances $C_{2}$ and $C_{3}$ to the receiver capacitance $C_{1}$, expressed by the mutual capacitances $C_{12}$ and $C_{13}$, respectively. The coupling between the transmitter capacitances is given by the mutual capacitance $C_{23}$. The corresponding coupling factors $k_{i j}(i, j=1,2,3)$ are defined by:

$$
k_{i j}=\frac{C_{i j}}{\sqrt{C_{i} C_{j}}}
$$

Resonance is created in each circuit by the inductors $L_{i}$ $(i=1,2,3)$ in parallel, with a value of

$$
L_{i}=\frac{1}{\omega_{0}^{2} C_{i}}
$$

In this work, we will provide the analytical solution for power maximization: we will determine the optimal load $Y_{1}$ to maximize the output power $P_{1}$ delivered to the load. 
For convenience, we introduce the following notations $(i, j=1,2,3)$ :

$$
\begin{array}{r}
x_{i j}=\omega_{0} C_{i j} \\
\chi_{i j}=\frac{x_{i j}}{\sqrt{g_{i i} g_{j j}}} \\
\theta=\sqrt{1+\chi_{12}^{2}+\chi_{13}^{2}}
\end{array}
$$

We consider the three-port network as defined in Fig. 1 with at the ports peak voltage phasors $V_{i}$ and peak current phasors $I_{i}(i=1,2,3)$. The three-port network is fully characterized by its admittance matrix $\boldsymbol{Y}_{\mathbf{0}}$ at frequency $\omega_{0}$. We can express the current-voltage relation of the three-port as $I=Y_{\mathbf{0}} . \boldsymbol{V}$, or:

$$
\left[\begin{array}{l}
I_{1} \\
I_{2} \\
I_{3}
\end{array}\right]=\left[\begin{array}{ccc}
g_{11} & -j x_{12} & -j x_{13} \\
-j x_{12} & g_{22} & -j x_{23} \\
-j x_{13} & -j x_{23} & g_{33}
\end{array}\right] \cdot\left[\begin{array}{l}
V_{1} \\
V_{2} \\
V_{3}
\end{array}\right]
$$

\section{B. Uncoupled transmitters}

We first consider the case where the transmitters are uncoupled, i.e. $x_{23}=0$. For this configuration, both transmitters function independently, and the entire system can be considered as the superposition of two separate CPT systems, each with one transmitter and one receiver. For this configuration, it was shown in [3] that the optimal load for power maximization is purely real, i.e. $B_{1}=0$. We thus obtain, with $I_{1}=-G_{1} V_{1}$ :

$$
\left[\begin{array}{c}
0 \\
I_{2} \\
I_{3}
\end{array}\right]=\left[\begin{array}{ccc}
g_{11}+G_{1} & -j x_{12} & -j x_{13} \\
-j x_{12} & g_{22} & 0 \\
-j x_{13} & 0 & g_{33}
\end{array}\right] \cdot\left[\begin{array}{c}
V_{1} \\
V_{2} \\
V_{3}
\end{array}\right]
$$

Inverting the $3 \times 3$ matrix, we find:

$$
V_{1}=\frac{g_{22} x_{13} I_{3}+g_{33} x_{12} I_{2}}{D} j
$$

with

$$
D=g_{11} g_{22} g_{33}+G_{1} g_{22} g_{33}+g_{22} x_{13}^{2}+g_{33} x_{12}^{2}
$$

The output power $P_{1}$ is given by [8]:

$$
P_{1}=\frac{1}{2} G_{1}\left|V_{1}\right|^{2}
$$

Substituting (8) in (10) and equalizing the partial derivative of (10) with regard to $G_{1}$ to zero, we find the optimal value for $G_{1}$ that maximizes power transfer:

$$
G_{1, o p t}=g_{11} \theta^{2}
$$

With this optimal load, the output power $P_{1}$ results in:

$$
P_{1, \max }=\frac{1}{8 \theta^{2}}\left[\frac{\chi_{12} I_{2}}{\sqrt{g_{22}}}+\frac{\chi_{13} I_{3}}{\sqrt{g_{33}}}\right]^{2}
$$

We notice that, if only one transmitter would be present (e.g, $x_{13}=\chi_{13}=0$ ), we obtain the same value for the optimal load and output power as derived by [3] for the configuration with a single transmitter and a single receiver.

\section{Coupled transmitters}

We now consider the case where the transmitters are coupled $\left(x_{23} \neq 0\right)$. We determine the Thévenin equivalent as seen from port 1 . The equivalent Thévenin voltage $V_{t h}$ can be found by calculating the open circuit voltage at port 1 , and thus by solving the system of equations given by (6) with $I_{1}=0$ and $V_{1}=V_{t h}$. We obtain

$$
V_{t h}=-\frac{x_{23}\left(x_{12} I_{3}+x_{13} I_{2}\right)-j\left(g_{22} x_{13} I_{3}+g_{33} x_{12} I_{2}\right)}{g_{11} g_{22} g_{33}\left(\theta^{2}+\chi_{23}^{2}\right)+2 j x_{12} x_{13} x_{23}}
$$

The equivalent Thévenin admittance $Y_{t h}$ can be computed by $Y_{t h}=I_{1} / V_{1}$ for the network where the current sources are replaced by an open circuit. By solving the system of equations given by (6) with $I_{2}=I_{3}=0$, we obtain:

$$
Y_{t h}=g_{1} \frac{\theta^{2}+\chi_{23}^{2}}{1+\chi_{23}^{2}}+j \frac{2 x_{12} x_{13} x_{23}}{g_{22} g_{33}+x_{23}^{2}}
$$

According to the maximum power transfer theorem, the optimal load $Y_{1, o p t}=G_{1, o p t}+j B_{1, o p t}$ at port 1 that realizes maximum power transfer is the complex conjugate of $Y_{t h}$. We obtain

$$
\begin{array}{r}
G_{1, o p t}=g_{11} \frac{\theta^{2}+\chi_{23}^{2}}{1+\chi_{23}^{2}} \\
B_{1, o p t}=-2 g_{11} \frac{\chi_{12} \chi_{13} \chi_{23}}{1+\chi_{23}^{2}}
\end{array}
$$

Notice that, contrary to the uncoupled configuration, a nonzero load susceptance is now necessary to realize power maximization.

From the Thévenin circuit loaded with $Y_{1, o p t}$, the maximum output power $P_{1, \max }$ can be calculated straightforwardly:

$$
\begin{aligned}
& P_{1, \max }=\left(\chi_{23}^{2}+\theta^{2}\right) . \\
& \frac{\left(\sqrt{g_{22}} \chi_{13} I_{3}+\sqrt{g_{33}} \chi_{12} I_{2}\right)^{2}+\chi_{23}^{2}\left(\sqrt{g_{22}} \chi_{12} I_{3}+\sqrt{g_{33}} \chi_{13} I_{2}\right)^{2}}{8 g_{22} g_{33}\left(1+\chi_{23}^{2}\right)\left[\left(\chi_{23}^{2}+\theta^{2}\right)^{2}+4 \chi_{12}^{2} \chi_{13}^{2} \chi_{23}^{2}\right]}
\end{aligned}
$$

For $\chi_{23}=0,(15),(16)$ and (17) reduce to the optimal load and output power for the uncoupled configuration.

We want to emphasize the similarity of our results with the dual configuration for inductive wireless power transfer [9].

\section{NUMERICAL VALIDATION}

We confirm the analytical derivation by circuit simulation in SPICE. Consider the network with two transmitters and one receiver of Fig. 1. We simulate two scenarios: the uncoupled and the coupled configuration, with the values given in Table I. Both scenarios are identical, except for the coupling $k_{23}$ between the transmitters.

For the uncoupled scenario, calculation from (11) results in the optimal load $G_{1, \text { opt }}^{\prime}$ for achieving maximum power transfer. Remember that the optimal load for the uncoupled configuration is purely resistive. We obtain $G_{1, \text { opt }}^{\prime}=1.6 \mathrm{mS}$. The corresponding output power $P_{1, \max }^{\prime}$ can be computed from (12): $P_{1, \max }^{\prime}=1.9 \mathrm{~W}$. 
TABLE I

SIMULATION VALUES FOR THE UNCOUPLED AND COUPLED CONFIGURATION. BOTH SCENARIOS ARE IDENTICAL, EXCEPT FOR THE COUPLING $k_{23}$ BETWEEN THE TRANSMITTERS.

\begin{tabular}{|l|c|c|}
\hline & Uncoupled & Coupled \\
\hline$f$ & $1.0 \mathrm{MHz}$ & $1.0 \mathrm{MHz}$ \\
$I_{2}$ & $200 \mathrm{~mA}$ & $200 \mathrm{~mA}$ \\
$I_{3}$ & $100 \mathrm{~mA}$ & $100 \mathrm{~mA}$ \\
$g_{11}$ & $1.0 \mathrm{mS}$ & $1.0 \mathrm{mS}$ \\
$g_{22}$ & $1.0 \mathrm{mS}$ & $1.0 \mathrm{mS}$ \\
$g_{33}$ & $2.0 \mathrm{mS}$ & $2.0 \mathrm{mS}$ \\
$C_{1}, C_{2}, C_{3}$ & $400 \mathrm{nF}$ & $400 \mathrm{nF}$ \\
$L_{1}, L_{2}, L_{3}$ & $63.3 \mu \mathrm{H}$ & $63.3 \mu \mathrm{H}$ \\
$k_{12}$ & $25 \%$ & $25 \%$ \\
$k_{13}$ & $25 \%$ & $25 \%$ \\
$k_{23}$ & $0 \%$ & $50 \%$ \\
\hline
\end{tabular}

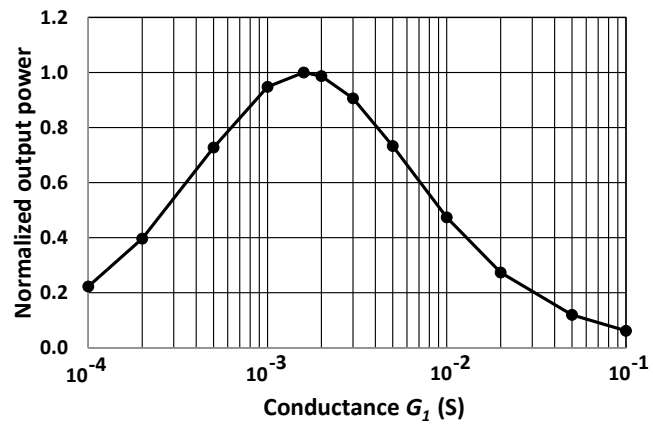

Fig. 2. The simulated output power, normalized to $P_{1, \max }^{\prime}=1.9 \mathrm{~W}$, for varying load conductance for the uncoupled scenario.

We first simulate the uncoupled scenario in SPICE for varying load conductance. Fig 2 shows the output power, normalized to $P_{1, \max }^{\prime}$. Notice that as well the value for the optimal load as for the output power correspond perfectly with the analytical derivation.

Secondly, consider the coupled scenario (Table I). The optimal load $G_{1, \text { opt }}^{\prime \prime}+j B_{1, \text { opt }}^{\prime \prime}$ now has a non-zero susceptance. We obtain $G_{1, o p t}^{\prime \prime}=1.3 \mathrm{mS}$ and $B_{1, o p t}^{\prime \prime}=-0.28 \mathrm{mS}$ from (15) and (16). The negative susceptance corresponds with an inductance of $574 \mu \mathrm{H}$. From (17), the maximum output power $P_{1, \max }^{\prime \prime}$ can be calculated: $P_{1, \max }^{\prime \prime}=1.1 \mathrm{~W}$. Fig. 3 shows the simulation results for the output power, normalized to $P_{1, \max }^{\prime \prime}$. The simulation results validate the analytical derived expressions.

Finally, we simulate the coupled scenario for varying load susceptance. Fig. 4 shows the output power, normalized to $P_{1, \max }^{\prime \prime}$, as function of varying load inductance. The load conductance is kept fixed at $G_{1, \text { opt }}^{\prime \prime}=1.3 \mathrm{mS}$. We notice that the optimal load inductance is given by the analytical derived value of $574 \mu \mathrm{H}$. Notice that for this example, the output power remains high for inductances larger than $G_{1, o p t}^{\prime \prime}$.

\section{CONCLUSION}

We analytically solved the power maximization problem for a CPT setup with two transmitters and a single receiver. If both transmitters are uncoupled, a purely resistive load applies. For coupled transmitters, a non-zero load susceptance is necessary

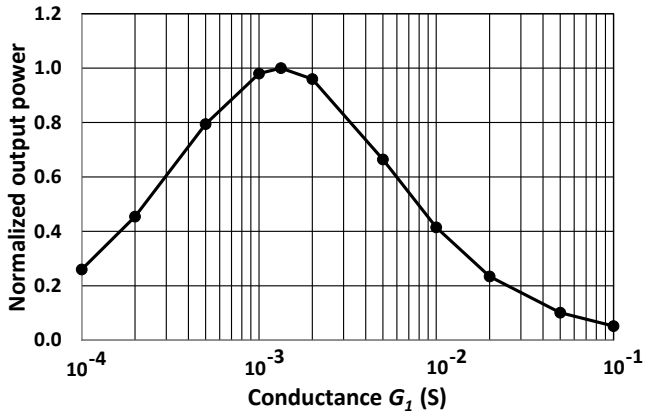

Fig. 3. The simulated output power, normalized to $P_{1, \max }^{\prime \prime}=1.1 \mathrm{~W}$, for varying load conductance for the coupled scenario. The load susceptance remains invariant and corresponds with an inductance of $574 \mu \mathrm{H}$.

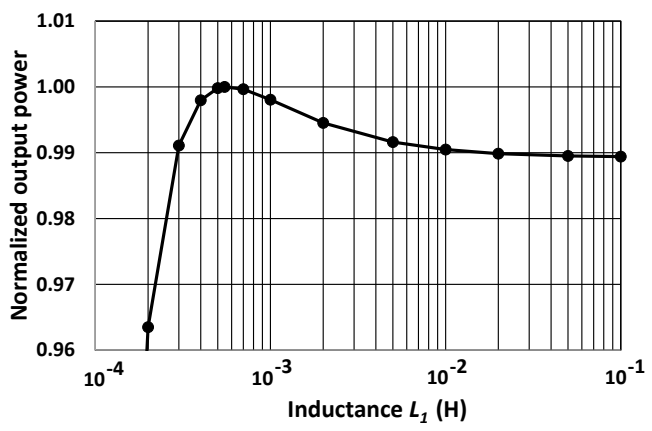

Fig. 4. The simulated output power, normalized to $P_{1, \max }^{\prime \prime}=1.1 \mathrm{~W}$, for varying load inductance for the coupled scenario. The load conductance remains fixed at $G_{1, \text { opt }}^{\prime \prime}=1.3 \mathrm{mS}$.

for power maximization. The analytical expressions were numerically validated by SPICE simulations.

\section{REFERENCES}

[1] Lu, F., Zhang, H. and Mi, C., 2017. A Review on the Recent Development of Capacitive Wireless Power Transfer Technology. Energies, 10(11), p.1752.

[2] Minnaert, B. and Stevens, N., 2017. Conjugate Image Theory Applied on Capacitive Wireless Power Transfer. Energies, 10(1), p.46.

[3] Dionigi, M., Mongiardo, M., Monti, G. and Perfetti, R., 2017. Modelling of wireless power transfer links based on capacitive coupling. International Journal of Numerical Modelling: Electronic Networks, Devices and Fields, 30(3-4).

[4] Minnaert, B. and Stevens, N., 2017. Optimal analytical solution for a capacitive wireless power transfer system with one transmitter and two receivers. Energies, 10(9), p.1444.

[5] Van Neste, C.W., Phani, A., Hull, R., Hawk, J.E. and Thundat, T., 2016, October. Quasi-wireless capacitive energy transfer for the dynamic charging of personal mobility vehicles. IEEE PELS Workshop on Emerging Technologies: Wireless Power Transfer (WoW), p. 196-199.

[6] Lu, F., Zhang, H., Hofmann, H., Mei, Y. and Mi, C., 2016, October. A dynamic capacitive power transfer system with reduced power pulsation. IEEE PELS Workshop on Emerging Technologies: Wireless Power Transfer (WoW), p. 60-64.

[7] Hong, J.S.G.; Lancaster, M.J. Microstrip filters for RF/microwave applications, 1st ed.; John Wiley \& Sons: New York, NY, USA, 2001; pp. 235-253.

[8] Montgomery, C.G.; Dicke, R.H.; Purcell, E.M. Principles of microwave circuits; McGraw-Hill Book Company: New York, NY, USA, 1948.

[9] Monti, G., Mongiardo, M., Wang, Q., Che, W. and Chang, Y., 2016, May. Wireless power transmission from two transmitters to one receiver: optimal design for power maximization. IEEE International Workshop on Electromagnetics: Applications and Student Innovation Competition (iWEM), p. 1-3. 